\title{
COMPUTER MODELING OF POINT DEFECTS, IMPURITY SELF-ORDERING EFFECTS AND SURFACES IN ADVANCED PEROVSKITE FERROELECTRICS
}

\author{
G. Borstel ${ }^{a}$, E.A. Kotomin ${ }^{a, b}$, R.I. Eglitis ${ }^{a}$ AND E. Heifets ${ }^{c}$ \\ ${ }^{a}$ Universität Osnabrück, Fachbereich Physik, 49069 Osnabrück, Germany \\ ${ }^{b}$ Institute of Solid State Physics, University of Latvia \\ Kengaraga str. 8, Riga 1063, Latvia \\ ${ }^{c}$ Materials and Processes Simulation Center, Beckman Institute \\ Caltech, Pasadena, CA 91125, USA
}

The calculated optical properties of basic point defects - F-type centers and hole polarons - in $\mathrm{KNbO}_{3}$ perovskite crystals are used for the interpretation of available experimental data. The results of quantum chemical calculations for perovskite $\mathrm{KNb}_{x} \mathrm{Ta}_{1-x} \mathrm{O}_{3}$ solid solutions are presented for $x=0$, $0.125,0.25,0.75$, and 1 . An analysis of the optimized atomic and electronic structure clearly demonstrates that several nearest $\mathrm{Nb}$ atoms substituting for $\mathrm{Ta}$ in $\mathrm{KTaO}_{3}$ - unlike Ta impurities in $\mathrm{KNbO}_{3}$ - reveal a self-ordering effect, which probably triggers the ferroelectricity observed in $\mathrm{KNb}_{x} \mathrm{Ta}_{1-x} \mathrm{O}_{3}$. Lastly, the (110) surface relaxations are calculated for $\mathrm{SrTiO}_{3}$ and $\mathrm{BaTiO}_{3}$ perovskites. The positions of atoms in 16 near-surface layers placed atop a slab of rigid ions are optimized using the classical shell model. Strong surface rumpling and surface-induced dipole moments perpendicular to the surface are predicted for both the O-terminated and Ti-terminated surfaces.

PACS numbers: 61.72.-y, 68.35.-p, 71.15.-m

\section{Introduction}

Most of real crystals are non-stoichiometric and thus contain along with impurities large concentrations of intrinsic defects - vacancies. Oxygen vacancies are known to give rise to $F^{+}$and $F$ centers (vacancy which trapped one or two electrons, respectively) $[1,2]$. The properties of $F$-type centers in ionic oxides like $\mathrm{MgO}$ and $\alpha-\mathrm{Al}_{2} \mathrm{O}_{3}$ are well studied, unlike $\mathrm{KNbO}_{3}$ where up to now there exists only a tentative assignment of the electron-induced absorption band at $2.7 \mathrm{eV}$ to the $F$-type centers [3]. In its turn, cation vacancies are able to trap radiation-induced holes. Thus, in irradiated $\mathrm{MgO}$ a cation vacancy is known to trap one or two holes giving rise to the $V^{-}$and $V^{0}$ centers $[2,4]$ called bound hole polaron and bipolaron, respectively. In the $V^{-}$center a hole is trapped by one of the $\mathrm{O}^{2-}$ ions 
neighboring to the cation vacancy, thus creating an $\mathrm{O}^{-}$paramagnetic ion, and a broad absorption band at $2.3 \mathrm{eV}$. The trapping of another hole on the opposite side of the vacancy results in a singlet spin state $(S=0)$ and a slightly perturbed absorption band.

Since the middle of the seventies a number of theoretical studies were performed which aimed at understanding the properties of hole centers in oxides. It was shown that the mentioned absorption band at $2.3 \mathrm{eV}$ in $\mathrm{MgO}$ corresponds to a small-polaron transition characterized by a hole transfer from a single $\mathrm{O}^{-}$to the equatorial $\mathrm{O}^{2-}$ neighboring ions, at a frozen lattice configuration. The key factor in a hole localization on a single $\mathrm{O}^{2-}$ ion lies in the asymmetric displacement of one of six (otherwise equivalent) oxygens from a regular lattice site. This displacement was calculated later using the classical shell model (SM) as implemented into the HADES code (the Harwell program HADES (Lidiard and Norgett (1972)). These calculations have, however, several limitations: (i) the assumption that a hole is entirely localized on a single oxygen ion; (ii) the short-range interaction between $\mathrm{O}^{-}$and surrounding ions is assumed to be the same as for a regular $\mathrm{O}^{2-}$ ion; (iii) a quantum mechanical treatment of a single $\mathrm{O}^{-}$ion does not allow to study directly the small-polaron transition of a hole to nearest $\mathrm{O}^{2-}$ ions. These limitations could be avoided by using larger quantum clusters with self-consistent treatment of the electronic/atomic structure and hole density distribution. For this purpose semi-empirical quantum chemical methods are well suited.

So far very little is done for understanding the hole polarons in perovskite $\mathrm{KNbO}_{3}$. Ti-doped $\mathrm{KNbO}_{3}$ shows an electron spin resonance (ESR) signal which was interpreted as a two-site polaron next to the impurity [5]. The relevant absorption band lies at $\approx 2 \mathrm{eV}$. Transient optical absorption spectra induced in $\mathrm{KNbO}_{3}$ by a pulsed electron beam reveal several bands, one of them is around $1 \mathrm{eV}$ [6]. Probably, this is caused by the $\mathrm{O}^{-}$bound polaron. No polarons associated with $\mathrm{K}$ vacancies were detected yet in this material but, similarly to $\mathrm{MgO}$, we expect such defects to exist. For example, in $\mathrm{BaTiO}_{3}$ hole polarons were found which are bound to $\mathrm{Na}$ or $\mathrm{K}$ ions replacing $\mathrm{Ba}$ and thus forming a negatively charged site attracting a hole. As to theoretical studies of defects in $\mathrm{KNbO}_{3}$, most of them deal mainly with defect formation energies calculated using a classical shell model (see Ref. [7] and references therein). In this paper we present results of electron and hole center calculations in $\mathrm{KNbO}_{3}$.

As the temperature decreases, $\mathrm{KNbO}_{3}$ goes through three ferroelectric phase transitions, whereas $\mathrm{KTaO}_{3}$ is only an incipient ferroelectric which becomes ferroelectric already at very low Nb impurity concentrations, $x \geq 0.01$. This raises the question about the nature of the phase transition in $\mathrm{KNb}_{x} \mathrm{Ta}_{1-x} \mathrm{O}_{3}$ (KTN) [8]. X-ray photoelectron spectroscopy (XPS) has shown [9] that Ta ions are replaced by the $\mathrm{Nb}$ ions. Additionally, X-ray absorption fine structure (XAFS) measurements [8] have demonstrated that the $\mathrm{Nb}$ sits in off-center position. Its [111] displacement is $0.145 \AA$ at $70 \mathrm{~K}$, and changes by less than 20 percent as the temperature increases to the room temperature. One of the purposes of this paper are therefore 
comparative calculations for $\mathrm{Nb}$ impurities in $\mathrm{KTaO}_{3}$ and vice versa, Ta in $\mathrm{KNbO}_{3}$, both for an isolated impurity and for clustered impurities in a cubic, paraelectric phase of these crystals.

Thin ferroelectric films are important for the development of optical waveguides and integrated optics applications. The $\mathrm{SrTiO}_{3}(110)$ surface is also used as a substrate for the cuprate high $T_{\mathrm{c}}$ superconductor growth. For these applications the atomic structure of the perovskite surfaces is very important [10-12]. This is why in this paper we calculate the atomic structure of $\mathrm{SrTiO}_{3}$ and similar $\mathrm{BaTiO}_{3}$ (110) surface in a cubic (paraelectric) phase of both crystals. (It should be reminded that at all temperatures bulk $\mathrm{SrTiO}_{3}$ exhibits paraelectric properties, whereas $\mathrm{BaTiO}_{3}$ undergoes phase transitions from paraelectric to ferroelectric as the temperature decreases.)

Recently several $a b$ initio studies were published for the (100) surface of these two crystals where a few near-surface planes were allowed to relax $[13,14]$. Due to many atomic coordinates to be calculated, such calculations are very complicated and time consuming. An alternative approach is to use a classical shell model (SM) [15]. The latter has been very successfully used for many years for defect calculations in the perovskite bulk [7]. The SM was very recently applied by us to the calculation of (100) surface relaxations for $\mathrm{BaTiO}_{3}[16]$ and $\mathrm{SrTiO}_{3}[17,18]$. We have discovered in these studies that approximately six near-surface planes are considerably disturbed as the surface is created, i.e. atoms are strongly displaced from their regular lattice sites. This results in the appearance of a dipole moment perpendicular to the surface, even in a cubic phase of perovskite crystals. The great advantage of SM is that it is well suited for the treatment of the polarization effects which are important for our study.

A comparison of our SM results [16-18] with the above-mentioned two $a b$ initio calculations $[13,14]$ and an experimental low-energy-electron diffraction (LEED) study for the $\mathrm{SrTiO}_{3}$ (001) surface [19] clearly demonstrates their good agreement for both the displacements of surface metal and oxygen atoms in opposite directions (the so-called rumpling) from their Ti-O perfect-plane sites and relative displacements of the second and third planes. This is a clear indication of the ability of the method which motivated us to perform calculations in this paper for the more advanced (110) surfaces of these crystals. The (110) surface was the subject of recent intensive experimental studies using scanning tuneling microscope (STM), ultraviolet photoelectron spectroscopy (UPS), and XPS spectroscopies [20] as well as Auger spectroscopy and LEED [21, 22]. The atomic and electronic structure of the (110) surface was found to be strongly dependent on the annealing temperature. We are not aware of any theoretical calculations of the $\mathrm{BaTiO}_{3}$ and $\mathrm{SrTiO}_{3}$ (110) surfaces. In this study we used an idealized model for a perfect and flat surface (see below). In particular, we focus on the rumpling effect since this is known to be quite considerable in many oxide crystals.

\section{Methods}

In calculations of the electronic structure of point defects we have used the semi-empirical, quantum chemical method of the intermediate neglect of the differ- 
ential overlap (INDO) [23]. This method is based on the Hartree-Fock formalism and allows for self-consistent calculations of the atomic and electronic structure of pure and defective crystals. The method has demonstrated very good results in previous calculations of defects, both in the bulk and on the surface, in many oxide materials [1], including pure $\mathrm{KNbO}_{3}$ and $\mathrm{KTaO}_{3}$ perovskites, and defects - $\mathrm{Li} \mathrm{im-}$ purities in $\mathrm{KTaO}_{3}[24,25]$. The calculated frequencies of the transverse-optic (TO) phonons at the $\Gamma$ point in the Brillouin zone (BZ) of cubic and rhombohedral $\mathrm{KNbO}_{3}$ and the atomic coordinates in the minimum energy configuration for the orthorhombic and rhombohedral phases of $\mathrm{KNbO}_{3}$ are also in good agreement with experiment, thus indicating that a very successful INDO parametrization has been achieved in Refs. [24, 25]. Frozen-phonon calculations for $T_{1 \mathrm{u}}$ and $T_{2 \mathrm{u}}$ modes of cubic $\mathrm{KTaO}_{3}$ are also in good agreement with experiment.

Covalency effects in the chemical bonding could be seen from the calculated (static) effective charges on atoms (calculated using the Löwdin population analysis): This gives $0.62 e$ for $\mathrm{K}, 2.23 e$ for $\mathrm{Ta}$ and $-0.95 e$ for $\mathrm{O}$ in $\mathrm{KTaO}_{3}$, which are far from those expected in the purely ionic model $(+1 e,+5 e$ and $-2 e$, respectively) often used. These charges show a slightly higher ionicity in $\mathrm{KTaO}_{3}$ as compared with the relevant effective charges calculated for $\mathrm{KNbO}_{3}$ : $0.54 e$ for $\mathrm{K}, 2.02 e$ for $\mathrm{Nb}$ and $-0.85 e$ for $\mathrm{O}$ atoms.

Along with our INDO method, the $a b$ initio calculations for perovskite KTN solid solutions were performed recently using the full-potential linear muffin-tin orbitals (FP-LMTO) code [26, 27]. The FP-LMTO calculations for $\mathrm{KNb}_{x} \mathrm{Ta}_{1-x} \mathrm{O}_{3}$ solid solutions have been performed using $2 \times 2 \times 2$ times extended supercells containing 40 atoms for $\mathrm{Nb}$ concentrations $x=0,0.25,0.75$, and 1 [28].

In terms of the SM, the interatomic interactions are described by the corecore, core-shell and shell-shell pair potentials. In this approach each ion has a charged core and electronic shell. The sum of the core and shell charges is equal to the formal charge of the corresponding ion. The spring constant $k$ connects the core and the shell of the same ion. The core-shell separation is a measure of the atomic polarization. The interactions between the cores and between cores and shells of different ions are Coulombic, whereas the interactions between the shells of different ions besides the Coulombic part contain also the short-range potentials accounting for the effects of the exchange repulsion together with the van-der-Waals attraction between them. The short-range Buckingham potentials contain three parameters $(A, \rho$, and $C)$ per pair of atoms. All parameters are carefully fitted to the lattice structure, elastic and dielectric properties of crystals (see details in Refs. $[7,15,17]$ ). The use of integer ionic charges does not imply restrictions to ionic materials, in fact the short-range potential effectively takes into account the covalency and charge-transfer effects. Atomistic surface relaxation is found by means of the MARVIN computer code [29]. This code effectively realizes the shell-model technique for simulations of the surface structures.

In our slab calculations we simulated both Ti- and O-terminated (110) surfaces. First, we have studied a periodic two-dimensional slab of cubic Sr- and $\mathrm{BaTiO}_{3}$. To this end, we have optimized the atomic positions in 16 near-surface 
planes, placed into the electrostatic field of the slab (simulated by 20 additional planes whose atoms were fixed in their perfect lattice sites). The number of these additional planes was chosen to reach a convergence of the crystalline field in the surface planes.
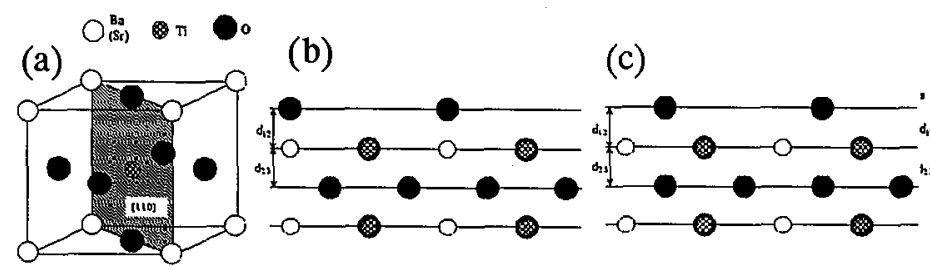

(d)

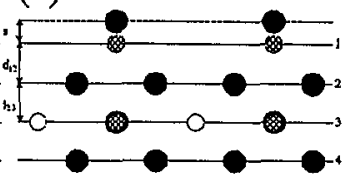

Fig. 1. Sketch of the cubic perovskite structure with shaded (110) surface (a), and definitions of the surface rumpling $s$ and the near-surface interplane distances $d_{12}$ and $d_{23}$ for the case of the O-terminated (b and c) and Ti-terminated (d) surfaces. In the case (b) $\mathrm{O}$ ions are initially displaced to the symmetrical positions in the direction parallel to the surface.

Figure 1 shows the sketch of the (110) surface and sequence of several planes below the surface. This demonstrates that the (110) surface could be either O-terminated or Ti-terminated. In the latter case $\mathrm{O}$ atoms can be displaced with respect to their Ti atom from the same ideal plane by the quantity $s$ (the rumpling). Atomic displacements from ideal planes also affect distances between several near-surface planes as compared to those in a perfect crystal. The relevant quantities $s, d_{12}, d_{23}$ shown in Fig. 1 could be measured experimentally, e.g., by means of LEED (as it was done for the (100) surface [19]). The main problem in the modeling of the (110) surface of $\mathrm{SrTiO}_{3}$ and $\mathrm{BaTiO}_{3}$ is that it consists of charged planes and if modeled as it appears after crystal cut, it has an infinite dipole moment perpendicular to the surface. To avoid this problem, in our calculations we removed half of $\mathrm{O}$ atoms from the Ti-terminated surface, and $\mathrm{Sr}(\mathrm{Ba})$ atoms from the Ti-terminated surface. As a result, we obtain a so-called type-II surface having no dipole moment. Since such a removal of half $O$ atoms from the surface disturbs the balance of interatomic interactions, there is no guarantee that half of the left $\mathrm{O}$ atoms do not want to displace in the direction parallel to the surface, additionally to the $z$ displacement - the only mode observed for the (100) surface. We studied this effect for the O-terminated surface of $\mathrm{SrTiO}_{3}$ (Fig. $1 \mathrm{~b}$ and $\mathrm{c}$ ).

\section{Main results and discussion \\ 3.1. Electronic structure of point defects}

Due to the above-mentioned considerable covalency of the chemical bonding in perovskites, it is not clear a priori how strongly a hole is localized on a single oxygen ion, as well as how many surrounding atoms are displaced. This is why it is important to perform comparative hole polaron simulations for both cubic crystals - highly ionic $\mathrm{MgO}$ and partly covalent $\mathrm{KNbO}_{3}$ - using quantum chemical calculations for big clusters or supercells. 


\subsubsection{Hole polarons}

The $\mathrm{MgO}$ calculations were done using 125 -atom clusters, whereas the polaron study for $\mathrm{KNbO}_{3}$ used 320 -atom supercells with periodically repeated defects. The optimized geometry of the $V^{-}$center in $\mathrm{MgO}$ shows that the $\mathrm{O}^{-}$ion, on which $80 \%$ of the hole density is localized, is displaced towards the cation vacancy by $3 \%$ of the interionic $\mathrm{Mg}-\mathrm{O}$ distance $(2.1 \AA)$. Displacements of other atoms are not significant. The effective charges of ions in a perfect crystal $( \pm 1.85 e)$ confirm the high ionicity of $\mathrm{MgO}$. The optical absorption energy of $2.2 \mathrm{eV}$ calculated by means of the delta self-consistent-field ( $\triangle \mathrm{SCF}$ ) method (the difference of two total self-consistent energies for the ground and excited states using the optimized ground state geometry) is very close to the experimental value (Table I). The thermal ionization energy necessary to release a hole to the valence band is found to be $1.6 \mathrm{eV}$.

\section{TABLE I}

Optical absorption $\left(E_{\mathrm{abs}}\right)$ and lattice relaxation $\left(E_{\mathrm{rel}}\right)$ energies calculated for hole centers in $\mathrm{MgO}$ and $\mathrm{KNbO}_{3}$, where ${ }^{a}$ stands for one-site polaron, ${ }^{b}$ for bi-polaron, and ${ }^{c}$ for two-site (molecular) polaron. FP-LMTO are results of the $a b$ initio method of full potential linearized muffin tin orbitals [30]. Experimental data for $\mathrm{MgO}[1,2]$ are given in brackets.

\begin{tabular}{c|c|c|c}
\hline \hline & \multirow{2}{*}{ Method } & \multicolumn{2}{|c}{ Energy [eV] } \\
\cline { 3 - 4 } & $E_{\text {abs }}$ & $E_{\text {rel }}$ \\
\hline $\mathrm{MgO}$ & INDO & $2.20(2.3)^{a}$ & $1.6(1.40)^{a}$ \\
& & $2.18(2.3)^{b}$ & $0.8(0.95)^{b}$ \\
\hline $\mathrm{KNbO}_{3}$ & INDO & $0.95^{a}$ & $0.40^{a}$ \\
& & $0.98^{c}$ & $0.53^{c}$ \\
\cline { 3 - 4 } & FP-LMTO & & $0.14^{a}$ \\
& & & $0.18^{c}$
\end{tabular}

For the $V^{0}$ center we found that the minimum energy corresponds to the two holes sitting on the opposite sides of the $\mathrm{Mg}$ vacancy, in agreement with experiment, which is accompanied by a $2 \%$ displacement of the two relevant $\mathrm{O}^{-}$ ions. The calculated absorption energy is close to that for the $V^{-}$center and to the experiment. Even more encouraging is the good agreement between the calculated thermal ionization energy of a second hole and the corresponding experimental value.

For hole polarons in $\mathrm{KNbO}_{3}$ we found two energetically favorable atomic configurations in which a hole is well localized; a sketch of the relevant one-site and two-site polarons is presented in Fig. 2. In the former case the $\mathrm{O}^{-}$ion is displaced towards the $\mathrm{K}$ vacancy by $3 \%$. Simultaneously, 11 other oxygens surrounding the 


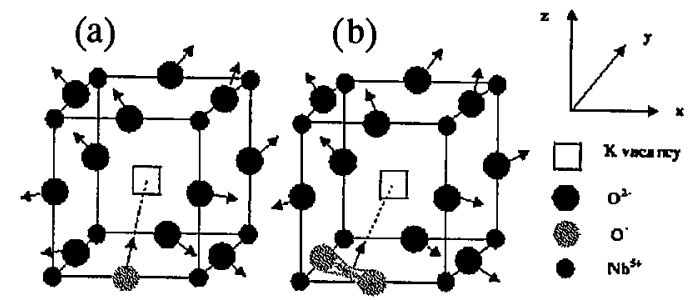

Fig. 2. Schematic view of the one-site (a) and two-site (b) hole polarons in $\mathrm{KNbO}_{3}$.

vacancy are slightly displaced outwards. The relevant lattice relaxation energy caused by atomic displacements is $0.4 \mathrm{eV}$ (Table I). In the one-site polaron the hole - like in $\mathrm{MgO}$ - is well localized by a single oxygen ion. The molecular orbital of the relevant local state contains a small contribution from atomic orbitals of other $\mathrm{O}$ ions, but not $\mathrm{K}$ or $\mathrm{Nb}$ ions, $80 \%$ of spin density is localized on the displaced $\mathrm{O}^{-}$atom. This is in contrast with properties of electronic $F$-type centers, where the ground state contains mainly the contribution from $\mathrm{Nb}$ atoms next to the $\mathrm{O}$ vacancy (see below). In agreement with the generally accepted polaronic model, the optical absorption energy of $\approx 1 \mathrm{eV}$ corresponds to a hole transfer to the state delocalized over nearest oxygens. In order to check the effect of interaction between periodically repeated polarons, we made also calculations for a one-site polaron using big quantum clusters. We found that the predicted optical absorption energy practically did not change.

In the two-site (molecular) polaron the two oxygens sharing a hole approach each other by $3.5 \%$ of the $\mathrm{O}-\mathrm{O}$ distance in a perfect lattice, and its center of mass is displaced towards the $\mathrm{K}$ vacancy by $2.2 \%$ (Fig. $2 \mathrm{~b}$ ). Other 10 nearest $\mathrm{O}$ ions are displaced outwards the $\mathrm{K}$ vacancy, like in the one-site hole case. The calculated absorption energy is quite close to that for one-site polaron (Table I) but twice smaller than the experimental value for a hole polaron trapped near a Ti impurity [5]. This shows that the optical absorption energy of small bound polarons can be strongly dependent on the type of defect involved [25]. The two-site polaron is lower in energy than the one-site polaron. This is in agreement with recent $a b$ initio FP-LMTO calculations [30], see also Table I.

\subsection{2. $F$ centers}

Preliminary INDO results on the $F$-type centers obtained using 40 atom supercells were presented in Refs. [31, 32]. Here we have used much larger 320-atom cells where the interaction between periodically repeated defects is small. In the $\mathrm{KNbO}_{3}$ cubic phase all $\mathrm{O}$ atoms are equivalent and have the local symmetry $C_{4 v}$ (due to which the excited state of the $F$-type centers could be split into a nondegenerate and a doubly-degenerate level). The optimized atomic relaxation around the $F$ center indicates the outward shift of the two Nb neighbors to the $\mathrm{O}$ vacancy by $3.9 \%$ which is associated with the lattice relaxation energy of $1.35 \mathrm{eV}$. This is considerably smaller than that for a 40 -atom supercell and quite close to the $a b$ initio FP-LMTO calculations (the $\mathrm{Nb}$ relaxation of $3.5 \%$ was accompanied by the energy gain of $0.5 \mathrm{eV}[32])$. 
The outward relaxation of nearest $K$ atoms and inward displacements of $\mathrm{O}$ atoms are much smaller. They give $\approx 20 \%$ of the net relaxation energy. The $F$ center local energy level lies $\approx 0.6 \mathrm{eV}$ above the top of the valence band. Its molecular orbital contains primarily the contribution from the atomic orbitals of the two nearest $\mathrm{Nb}$ atoms. Only $\approx 0.6 e$ resides at the orbitals centered at the vacancy site, with the same amount of electron density localized at the two nearest $\mathrm{Nb}$ atoms. Hence the electron localization at the vacancy is much smaller than the one known for $F$ centers in ionic oxides [1]. This difference could be due to the fact that the electrostatic (Madelung) field potential on a $\mathrm{O}$ site in $\mathrm{KNbO}_{3}$ is much smaller than that in ionic $\mathrm{MgO}(10.47 \mathrm{eV}$ vs. $23 \mathrm{eV})$ and an electron is respectively less bound.

For the charged $F^{+}$center the relaxation energy of $2.23 \mathrm{eV}$ and the $\mathrm{Nb}$ displacements of $5.1 \%$ are larger than those for the $F$ center due to a stronger Coulomb repulsion between unscreened $\mathrm{O}$ vacancy and $\mathrm{Nb}$ atoms: the portion of the electron density inside the $\mathrm{O}$ vacancy decreases to $0.3 e$.

The INDO calculated optical absorption energies for the $F^{+}$and $F$ centers are $2.30,2.63 \mathrm{eV}$ and $2.68,2.93 \mathrm{eV}$, respectively. That is, both defects are predicted to have one of the bands around 2.6-2.7 eV, in agreement with the experimental observation [3]. Thus, ESR experiments are necessary to discriminate between $F^{+}$ and $F$ centers in $\mathrm{KNbO}_{3}$.

\subsection{KTN solid solutions}

Figure $3 \mathrm{a}$ shows the total energy for $\mathrm{Nb}$ impurity concentrations at $x=0$, $0.125,0.25,0.75$, and 1 in $\mathrm{KNb}_{x} \mathrm{Ta}_{1-x} \mathrm{O}_{3}$ solid solution as a function of $\mathrm{Nb}$ [111] and [100] off-center displacements calculated by means of the INDO method. The conclusion can be drawn that the INDO Nb displacement of $0.146 \AA$ is very close to the experimental XAFS finding at $70 \mathrm{~K}[8]$, which corresponds to a $\mathrm{Nb}$ impurity concentration as low as $x=0.125$. Figure $3 \mathrm{~b}$ shows that similar FP-LMTO [28] calculations can reproduce experimental XAFS findings regarding $\mathrm{Nb}$ impurity off-center displacement only when $\mathrm{Nb}$ impurity concentrations in KTN reach $x=0.75$, on the contrary to experiments [8].

For the exploration of the $\mathrm{Nb}$ clusters in $\mathrm{KTaO}_{3}$, we extended our primitive $\mathrm{KTaO}_{3}$ unit cell $4 \times 4 \times 4$, i.e. 64 times, which is equivalent to a band structure calculation at $64 k$ points in the Brillouin zone. Then we replaced in our extended unit cell, containing 320 atoms, seven Ta atoms by seven $\mathrm{Nb}$ atoms (see Fig. 4). In order to find the energy minimum for a seven- $\mathrm{Nb}$ ion cluster in $\mathrm{KTaO}_{3}$, we allowed the six $\mathrm{Nb}$ atoms to relax towards the central $\mathrm{Nb}$ atom. The positions of the $\mathrm{K}$ and $\mathrm{O}$ atoms were kept fixed. The results of our INDO calculations show (Fig. 5) that the total energy per large unit cell is lowered by $0.088 \mathrm{eV}$ when the six $\mathrm{Nb}$ atoms shift symmetrically by $0.187 \AA$ towards the central Nb atom. Moreover, a uniform outward displacement of the six $\mathrm{Nb}$ atoms from the central $\mathrm{Nb}$ (see Fig. 4) by $0.073 \AA$ is also favorable and lowers the energy by approximately $0.03 \mathrm{eV}$ (Fig. 5). In the case, when the six $\mathrm{Nb}$ atoms are shifted outwards from the central $\mathrm{Nb}$ atom, but the latter moves off-center by $0.27 \AA$ in the [111] direction, a further lowering of $0.09 \mathrm{eV}$ is found to give an overall total energy lowering of approximately $0.12 \mathrm{eV}$. 
(a)

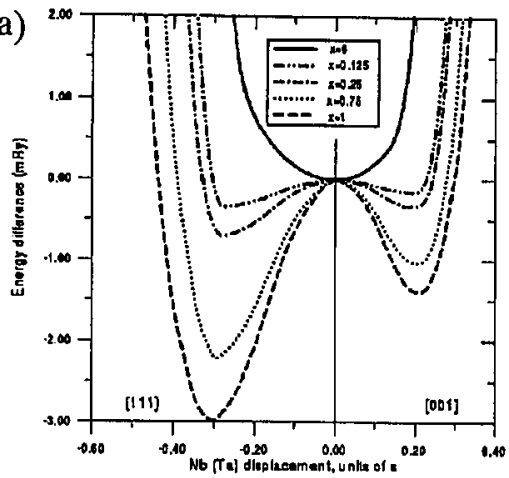

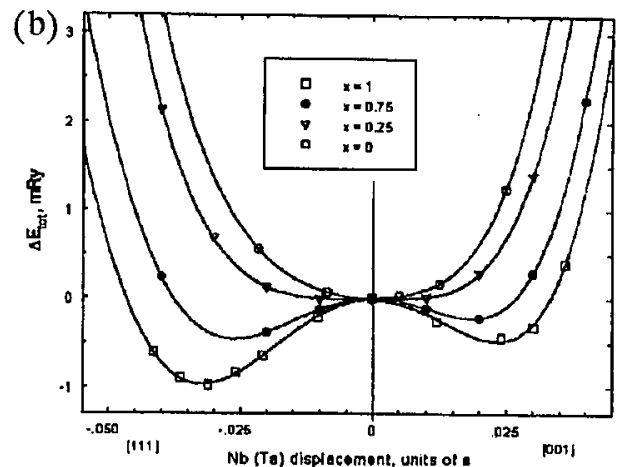

Fig. 3. The difference in total energy due to $\mathrm{Nb}$ off-center [111] and [100] displacement in $\mathrm{KNb}_{x} \mathrm{Ta}_{1-x} \mathrm{O}_{3}$ solid solution for $\mathrm{Nb}$ concentrations of $x=0,0.125,0.25,0.75$, and 1 , as calculated by means of INDO (a) and FP-LMTO methods [28] (b).

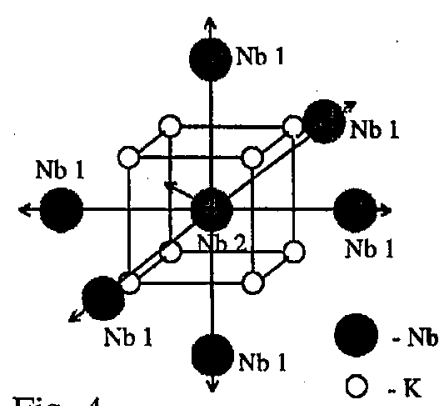

Fig. 4

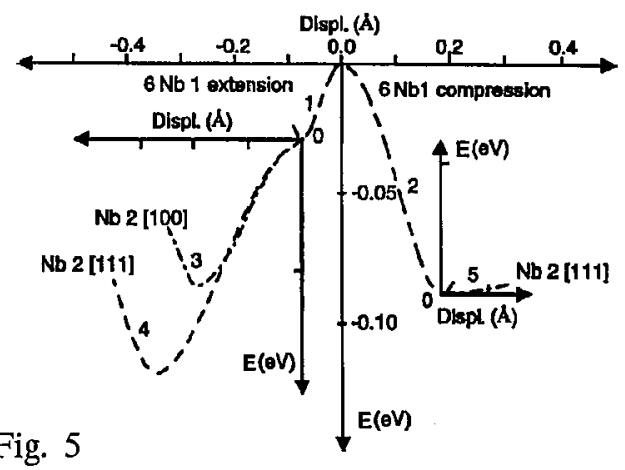

Fig. 4. Sketch of an asymmetric relaxation in a $\mathrm{Nb}$ cluster containing seven nearest-neighbor $\mathrm{Nb}$ atoms in $\mathrm{KTaO}_{3}$, calculated using a $4 \times 4 \times 4$ extended unit cell, containing 320 atoms; six Nb 1 atoms relax outwards whereas the central $\mathrm{Nb} 2$ atom is off-center displaced along the [100] or [111] direction.

Fig. 5. Total energy vs. displacement of six $\mathrm{Nb}$ atoms in $\mathrm{KTaO}_{3}$ which are relaxed outwards (curve 1) and inwards (curve 2) with respect to the center of the seven- $\mathrm{Nb}$ atom cluster. The central $\mathrm{Nb}$ atom prefers an off-center position in the [100] and [111] directions (curves 3 and 4, respectively). In the case of symmetrical inward compression of impurity cluster it remains on center (curve 5).

The central $\mathrm{Nb}$ atom reveals also an instability in the [100] direction, with a shift of $0.192 \AA$ with an additional energy lowering of $0.056 \mathrm{eV}$ after the six $\mathrm{Nb}$ atoms were shifted outwards from the central $\mathrm{Nb}$ atom. The central $\mathrm{Nb}$ atom exhibits an on-center behavior (Fig. 5) in the case when the six $\mathrm{Nb}$ atoms move towards the central $\mathrm{Nb}$ atom.

We have also studied the opposite case and found that when seven Ta impurities replace seven $\mathrm{Nb}$ atoms in $\mathrm{KNbO}_{3}$, a qualitatively different behavior is observed compared to the case, when seven $\mathrm{Nb}$ atoms replace seven Ta atoms in $\mathrm{KTaO}_{3}$. The total energy minimum of the former case corresponds to a symmet- 
rically extended $(0.04 \AA)$ configuration of the six Ta ions, while the central Ta ion retains its on-center position.

\subsection{Perfect surface relaxation}

The calculated displacements of cores and shells of ions in several top layers nearby the (110) surface will be discussed in detail in a separate paper. Table II based on these data gives our predictions for the surface rumpling and the relative displacements of the two top layers. For the Ti-terminated surface the surface rumpling for both $\mathrm{SrTiO}_{3}$ and $\mathrm{BaTiO}_{3}$ (110) surfaces is similar and very large, $\approx 14 \%$ (hereafter all displacements are in units of the bulk lattice parameter). This arises from a combination of a strong $O$ ion outward displacement $(\approx 8 \%)$ and a $\mathrm{Ti}$ ion inward displacement (by $6 \%$ ). This relaxation is much larger than the one we found earlier for the (100) surface. The reduction of relative distances between the first three layers is quite similar for both crystals, $4-5 \%$.

\section{TABLE II}

Surface rumpling $s$, and relative displacements of the three-near surface planes (see Fig. 1) for the $O$ - and Ti-terminated $\mathrm{SrTiO}_{3}$ and $\mathrm{BaTiO}_{3}(110)$ surfaces (in percent of the bulk lattice parameter). Numbers in brackets are for the case when the surface $\mathrm{O}$ atoms are placed initially into positions shown in Fig. 1c, and move further in the direction parallel to the surface.

\begin{tabular}{c|c|c|c|c|c}
\hline \hline & \multicolumn{2}{|c|}{ O-termination } & \multicolumn{3}{|c}{ Ti-termination } \\
\cline { 2 - 6 } & $\delta d_{12}$ & $\delta d_{23}$ & $s$ & $\delta d_{12}$ & $\delta d_{23}$ \\
\hline $\mathrm{SrTiO}_{3}$ & 2.36 & -2.73 & 14.47 & -4.27 & -3.86 \\
& $(-11.83)$ & $(8.69)$ & & & \\
\hline $\mathrm{BaTiO}_{3}$ & 3.34 & -3.89 & 13.38 & -5.27 & -3.25
\end{tabular}

For another, O-terminated surface when $\mathrm{O}$ ions sit in symmetric positions (Fig. 1b), they are displaced only along the $z$ axis perpendicular to the surface. $\mathrm{O}$ atoms slightly move inwards by $\approx 3 \%$ in both crystals. Ti ions in the second plane also do so, which results in a relatively small decrease in the $d_{12}$ distance. This is, however, accompanied by a very large, 27-30\%, outward displacement of $\mathrm{Sr}(\mathrm{Ba})$ ions from the second plane. If $\mathrm{O}$ ions in $\mathrm{SrTiO}_{3}$ are placed initially into asymmetric positions and allowed to displace also in the direction parallel to the surface (Fig. 1c), we found another surface structure. In this structure Sr atoms in the second plane are only moderately $(\approx 4 \%)$ shifted outwards, but now the surface $\mathrm{O}$ ions tend to approach $\mathrm{Ti}$ ions in the second layer and move inwards so that they are displaced along the $\mathrm{z}$ axis as much as $14 \%$. The surface $\mathrm{O}$ ions are also strongly polarized, the relative core-shell separation is $\approx 4 \%$. As a result, the first and second planes turn out to be strongly compressed, their separation is reduced by $\approx 12 \%$, whereas the distance between the second and third planes 
increases by $\approx 9 \%$ due to a strong outward displacement of $O$ atoms $(-11 \%)$ in the third plane.

We calculated also the surface dipole moments as a function of a number of relaxed near-surface layers and found that they strongly oscillate as the number of relaxed near-surface layers increases from one to ten. For a larger number of relaxed layers these oscillations practically vanish and the surface dipole moment saturates. In other words, relaxation of ten top layers is important for a correct description of real (110) surfaces which imposes strict restrictions for the use of $a b$ initio methods. The calculated values of the surface dipole moments saturate at $1.12 \mathrm{e} \cdot \AA$ for O-terminated $\mathrm{SrTiO}_{3}$ and $-1.58 \mathrm{e} \cdot \AA$ for the Ti-terminated surface. This is in sharp contrast to our results for the (100) surface where we got much smaller dipole moments of $-0.167 \mathrm{e} \cdot \AA$ in the Sr-terminated case and $-0.447 \mathrm{e} \cdot \AA$ for the Ti-terminated surface. That is, for the (110) surface dipole moments and respectively, surface polarizations, are much larger and have opposite signs for the two terminations. The importance of the surface relaxation is seen also from the large decrease in the surface energy as a result of relaxation.

\section{Summary}

Based on our INDO calculations of the optical properties of the $F$ centers and hole polarons, we confirm their tentative experimental assignments $[3,6]$. We would like to stress that our numerical INDO calculations for KTN solid solutions have demonstrated that a single $\mathrm{Nb}$ impurity at a relative concentration of $x=0.125$ in $\mathrm{KTaO}_{3}$ reveals an off-center displacement which is close to that found in XAFS [8] experiments. Our INDO calculations allow a better reproduction of experimental XAFS findings than early FP-LMTO calculations [28]. According to the latter, $\mathrm{Nb}$ impurities in KTN become off-center only at a Nb impurity concentration of $x=0.75$.

Close Nb impurities in KTN are self-ordered and reveal a cooperative behavior. This probably triggers the KTN ferroelectric phase transition. In contrast, Ta impurities in $\mathrm{KNbO}_{3}$ reveal no self-ordering. Qualitatively different behavior of $\mathrm{Nb}$ and Ta atoms could be caused by a tiny difference in their chemical bonding with other atoms, first of all oxygens. A comparative analysis of the effective charges show that the charge for a single Ta impurity in $\mathrm{KNbO}_{3}$ is $2.15 e$ whereas that of a host $\mathrm{Nb}$ atom is 2.02e. This confirms our conclusion [25] about a higher ionicity of $\mathrm{Ta}$ in comparison with $\mathrm{Nb}$ atoms in pure crystals. In its turn, the effective charge of a single $\mathrm{Nb}$ impurity in $\mathrm{KTaO}_{3}$ is $2.08 e$ (whereas for the host Ta atoms it is $2.23 e$ ). The effective charge of $\mathrm{Ta}$ ions in a seven-ion cluster in $\mathrm{KNbO}_{3}$ is $2.18 e$, and that of $\mathrm{Nb}$ ion in a seven-ion cluster in $\mathrm{KTaO}_{3}$ is $2.06 e$, again smaller.

To summarize, we have demonstrated in this paper that modern large-scale computer modeling of complicated advanced materials is a very efficient tool for understanding their properties. In particular, we found that surface relaxation for $\mathrm{SrTiO}_{3}$ and $\mathrm{BaTiO}_{3}$ (110) surfaces could be much larger than that for the (100) surface. We predict also the possibility of two quite different surface relaxations for the O-terminated surface. Of great interest is to check these results experimentally by means of LEED and/or careful ab initio calculations. The formation of large 
dipole moments perpendicular to the surface, even in a cubic phase of a perovskite, which we observed earlier also for the relaxed (100) surface, can considerably affect the ferroelectric properties of thin films.

\section{Acknowledgments}

E.A.K. was partly supported by the Latvian National Programme on Microand Optoelectronics and by the DAAD. G.B. and R.E. thank the Deutsche Forschungsgemeinschaft for financial support within the SFB 225. The authors are indebted to A.V. Postnikov, A.M. Stoneham and O.F. Schirmer for many stimulating discussions.

\section{References}

[1] E.A. Kotomin, A.I. Popov, Nucl. Instrum. Methods B 141, 1 (1998).

[2] Y. Chen, M.M. Abraham, J. Phys. Chem. Solids 51, 747 (1990).

[3] E.R. Hodgson, C. Zaldo, F. Agullo-Lopez, Solid State Commun. 75, 351 (1990).

[4] A.E. Hughes, B. Henderson, in: Point Defects in Solids, Eds. J.H. Crawford Jr., L.M. Slifkin, Plenum Press, New York 1972, ch. 4.

[5] E. Possenriede, B. Hellermann, O.F. Schirmer, Solid State Commun. 65, 31 (1988).

[6] L. Grigorjeva, D. Millers, E.A. Kotomin, S. Polzik, Solid State Commun. 104, 327 (1997).

[7] M.J. Akhtar, Z.N. Akhtar, R.A. Jackson, C.R.A. Catlow, J. Am. Ceram. Soc. 78, 421 (1995).

[8] O. Hanske-Petitpierre, Y. Yacoby, J. Mustre de Leon, E.A. Stern, J.J. Rehr, Phys. Rev. B 44, 6700 (1991).

[9] R. Niemann, H. Hartmann, B. Schneider, H. Hesse, M. Neumann, J. Phys., Condens. Matter 8, 5837 (1996).

[10] Photorefractive Materials and their Applications, Eds. P. Günter, J.-P. Huignard, in series Topics in Applied Physics, Vol. 61, 62, Springer-Verlag, Berlin 1988.

[11] M.E. Lines, A.M. Glass, Principles and Applications of Ferroelectrics and Related Materials, Clarendon, Oxford 1977.

[12] O. Auciello, J.F. Scott, R. Ramesh, Physics Today, July 22, 1998.

[13] J. Padilla, D. Vanderbilt, Surf. Sci. 418, 64 (1998).

[14] J. Padilla, D. Vanderbilt, Phys. Rev. B 56, 1625 (1997).

[15] Computer Simulations of Solids, Eds. C.R.A. Catlow, W.C. Mackrodt, in series Lecture Notes in Physics, Vol. 166, Springer-Verlag, Berlin 1982.

[16] E. Heifets, S. Dorfman, D. Fuks, E.A. Kotomin, Thin Solid Films 296, 76 (1997).

[17] E. Heifets, S. Dorfman, D. Fuks, E.A. Kotomin, A. Gordon, J. Phys., Condens. Matter 10, L347 (1998).

[18] S. Dorfman, D. Fuks, E.A. Kotomin, Thin Solid Films 318, 65 (1998).

[19] N. Bickel, G. Schmidt, K. Heinz, K. Müller, Phys. Rev. Lett. 62, 2009 (1989).

[20] H. Bando, Y. Aiura, Y. Haruyama, T. Shimizu, Y. Nishihara, J. Vac. Sci. Technol. $B$ 13, 1150 (1995). 
[21] J. Brunen, J. Zegenhagen, Surf. Sci. 389, 349 (1997).

[22] J. Zegenhagen, T. Haage, Q.D. Jiang, Appl. Phys. A 67, 711 (1998).

[23] J.A. Pople, D.L. Beveridge, Approximate Molecular Orbital Theory, McGraw-Hill, New York 1970.

[24] R.I. Eglitis, A.V. Postnikov, G. Borstel, Phys. Rev. B 54, 2421 (1996).

[25] R.I. Eglitis, A.V. Postnikov, G. Borstel, Phys. Rev. B 55, 12976 (1997).

[26] M. Methfessel, Phys. Rev. B 38, 1537 (1988).

[27] M. Methfessel, C.O. Rodriguez, O.K. Andersen, Phys. Rev. B 40, 2009 (1989).

[28] A.V. Postnikov, T. Neumann, G. Borstel, Ferroelectrics 164, 101 (1995).

[29] D.H. Gay, A.L. Rohl, J. Chem. Soc. Faraday Trans. 91, 925 (1995).

[30] E.A. Kotomin, R.I. Eglitis, A.V. Postnikov, G. Borstel, N.E. Christensen, Phys. Rev. $B$ 60, 1 (1999).

[31] E.A. Kotomin, R.I. Eglitis, A.I. Popov, J. Phys., Condens. Matter 9, L315 (1997).

[32] R.I. Eglitis, N.E. Christensen, E.A. Kotomin, A.V. Postnikov, G. Borstel, Phys. Rev. B 56, 8599 (1997). 